\title{
The Study of Symmetry Energy in Pasta of Neutron Star From Compressible Liquid Drop Model Approximation
}

\author{
Eko Tri Sulistyani ${ }^{1}$, Rizky Ananda ${ }^{2}$ \\ ${ }^{1}$ Astrophysics Research Group, ${ }^{2}$ Physics Department, Faculty of Mathematics and Science, Universitas Gadjah Mada \\ Jl Bulaksumur, Yogyakarta, 55281, Indonesia. Tel. +62-274-6492599, Fax. +62 -274- 565223 \\ ${ }^{2}$ Email: rizky.ananda@mail.ugm.ac.id
}

\begin{abstract}
The properties of pasta which is located at the bottom of inner crust from neutron star has been studied by using compressible liquid drop model. Compressible liquid drop model is a modified liquid drop model as a density function. Liquid drop model based on assumption that the magnitude of nucleus bonding energy is contribution of surface, Coulomb, volume, symmetry, and proton-neutron pair effect. Pasta of neutron star behaves like liquid crystals (mesomhorpic phase). The top layer of pasta filled by free neutron gas, while in the lowest layer of the pasta is filled by proton-neutron gas. The properties of pasta are observed at temperatures close to zero Kelvin with the assumption that neutron star is on ground state and non accretion. The study of pasta emphasizes on symmetry energy's influence. Symmetry energy reduces the magnitude of bonding energy of nucleon in the nucleus and it causes nucleon to be more easily released from nucleus. After that, symmetry energy influence the properties of pasta, such as the shape of nucleus that is non spherical (some like plates, rods, and bubbles), the fluctuative values of Wigner-Seitz cell, and uneven distribution of protons and neutrons in the pasta region of neutron star.
\end{abstract}

Keywords: Compressible liquid drop model, Neutron star, Pasta, Symmetry energy

\section{INTRODUCTION}

The existence of neutron star was first discovered by thermal radiation from the surface of an isolated neutron star that was cooling down captured by a detector installed on a rocket when it exited from Earth's atmosphere. In 1967, astronomer Jocelyn Bell discovered well radio signals through radio wave telescopes. The object named pulsar (pulsating radio sources). A year after publication of Jocelyn, Gold proposed pulsars are rotating neutron stars at very high densities which causes the gravitational field is too strong (Yasrina, 2011).

Called neutron stars because it contains of abundance neutrons, mainly in the core of star. The main difference between neutrons in the nucleus and neutrons in neutron stars is atomic nucleus is only bound by strong nuclear forces, whereas in neutron stars, nuclear forces are also bound by gravitational forces. The gravitational binding energy of a neutron star is about $10 \%$ of neutron star's mass, while the bonding energy of a nucleus by $\mathrm{Fe}^{56}$ is $9 \mathrm{MeV} /$ nucleon, equivalent to $1 \%$ of the mass of $\mathrm{Fe}^{56}$ (Glendenning, 2000).

Neutron star has a mass $M \sim(1-2) \mathrm{M}_{\odot}$ and has radius of $R \approx(10-14) \mathrm{km}$. The maximum mass that can be possessed by a neutron star is $M \sim 1.5 M_{\odot}$ and its radius $R \sim 3 \mathrm{~km}$. Basically size of a neutron star depends on equilibrium of gravitational force with the pressure of degenerated neutron. The density of neutron stars is $\rho \simeq(2-3) \rho_{o}$, where $\rho_{o}=2,8 \times 10^{14} \mathrm{gr} \mathrm{cm}^{-3}$ is normal density of nucleus. Its gravitational energy is $E_{\text {grav }} \sim G M^{2} / R \sim 5 \times 10^{53} \mathrm{erg} \sim 0,2 M c^{2}$, and the gravity on its surface is $g \sim G M R^{2} \sim 2 \times 10^{14} \mathrm{~cm} \mathrm{~s}^{-2}\left(2 \times 10^{11}\right.$ times than Earth) (Haensel et al., 2007).

The structure of neutron star can be subdivided into the atmosphere, ocean, outer crust, inner crust and mantle. The outer crust consists of plasma ions and ionized electrons (Potekhin, 2011). A very thin surface layer (up to few meters in a hot star), contains a nondegenerate electron gas. In deeper layers the electrons a strongly degenerate, almost ideal gas, which becomes ultrarelativistic $\rho \gg 10^{6} \mathrm{gr} \mathrm{cm}^{-3}$ (Haensel et al., 2007). The pressure is mainly provided by electrons. The matter of inner crust consists of electrons, free neutrons $n$, and neutron-rich atomic nuclei. The fraction of free neutrons increases with growing mass density. The pressure of inner crust is produced by degenerated neutrons (Potekhin, 2011).

Pasta of neutron stars is a region that has properties such as liquid crystals, it's located in transition region of inner crust to outer core and called "mantle". In mantle region, special properties are found in the form of non-spherical atomic nuclei, such as rods, plates, tubes and bubbles. In addition, at pasta are found ocean of free neutrons gas in the inner crust region, with form of nuclei like rods and plates. Also found areas containing oceans of protons and neutrons when nucleus shape like a tube and bubble, more precisely it's found in the region closed to outer core.

For knowing the structure and state of neutron stars can't be execute directly. The observations to neutron stars are only based on emitted spectrum with the results in the form of luminosity and spectrum energy (frequency and wavelength) to determine the mass, radius, and surface temperature of neutron stars in 
general. According to observational data, it is known that neutron star has a mass interval $1.2 \mathrm{M} \odot$ to $2 \mathrm{M}_{\odot}$, and radius interval is $10 \mathrm{~km}$ to $14 \mathrm{~km}$. These results obtained in accordance with computational calculations for theoretical models. Therefore, theoretical models built to describe the structure and state of neutron stars can be used to study the state and structure of neutron stars (Haensel et al., 2007).

The existence of neutron star was first discovered by thermal radiation from the surface of an isolated neutron star that was cooling down captured by a detector installed on a rocket when it exited from Earth's atmosphere. In 1967, astronomer Jocelyn Bell discovered well radio signals through radio wave telescopes. The object named pulsar (pulsating radio sources). A year after publication of Jocelyn, Gold proposed pulsars are rotating neutron stars at very high densities which causes the gravitational field is too strong (Yasrina, 2011).

Called neutron stars because it contains of abundance neutrons, mainly in the core of star. The main difference between neutrons in the nucleus and neutrons in neutron stars is atomic nucleus is only bound by strong nuclear forces, whereas in neutron stars, nuclear forces are also bound by gravitational forces. The gravitational binding energy of a neutron star is about $10 \%$ of neutron star's mass, while the bonding energy of a nucleus by $\mathrm{Fe}^{56}$ is $9 \mathrm{MeV} /$ nucleon, equivalent to $1 \%$ of the mass of $\mathrm{Fe}^{56}$ (Glendenning, 2000).

Neutron star has a mass $M \sim(1-2) \mathrm{M}_{\odot}$ and has radius of $R \approx(10-14) \mathrm{km}$. The maximum mass that can be possessed by a neutron star is $M \sim 1.5 M_{\odot}$ and its radius $R \sim 3 \mathrm{~km}$. Basically size of a neutron star depends on equilibrium of gravitational force with the pressure of degenerated neutron. The density of neutron stars is $\rho \simeq(2-3) \rho_{o}$, where $\rho_{o}=2,8 \times 10^{14} \mathrm{gr} \mathrm{cm}^{-3}$ is normal density of nucleus. Its gravitational energy is $E_{\text {grav }} \sim G M^{2} / R \sim 5 \times 10^{53} \mathrm{erg} \sim 0,2 M c^{2}$, and the gravity on its surface is $g \sim G M R^{2} \sim 2 \times 10^{14} \mathrm{~cm} \mathrm{~s}^{-2}\left(2 \times 10^{11}\right.$ times than Earth) (Haensel et al., 2007).

The structure of neutron star can be subdivided into the atmosphere, ocean, outer crust, inner crust and mantle. The outer crust consists of plasma ions and ionized electrons (Potekhin, 2011). A very thin surface layer (up to few meters in a hot star), contains a nondegenerate electron gas. In deeper layers the electrons a strongly degenerate, almost ideal gas, which becomes ultrarelativistic $\rho \gg 10^{6} \mathrm{gr} \mathrm{cm}^{-3}$ (Haensel et al., 2007). The pressure is mainly provided by electrons. The matter of inner crust consists of electrons, free neutrons $n$, and neutron-rich atomic nuclei. The fraction of free neutrons increases with growing mass density. The pressure of inner crust is produced by degenerated neutrons (Potekhin, 2011).

Pasta of neutron stars is a region that has properties such as liquid crystals, it's located in transition region of inner crust to outer core and called "mantle". In mantle region, special properties are found in the form of non-spherical atomic nuclei, such as rods, plates, tubes and bubbles. In addition, at pasta are found ocean of free neutrons gas in the inner crust region, with form of nuclei like rods and plates. Also found areas containing oceans of protons and neutrons when nucleus shape like a tube and bubble, more precisely it's found in the region closed to outer core.

For knowing the structure and state of neutron stars can't be execute directly. The observations to neutron stars are only based on emitted spectrum with the results in the form of luminosity and spectrum energy (frequency and wavelength) to determine the mass, radius, and surface temperature of neutron stars in general. According to observational data, it is known that neutron star has a mass interval $1.2 \mathrm{M}_{\odot}$ to $2 \mathrm{M}_{\odot}$, and radius interval is $10 \mathrm{~km}$ to $14 \mathrm{~km}$. These results obtained in accordance with computational calculations for theoretical models. Therefore, theoretical models built to describe the structure and state of neutron stars can be used to study the state and structure of neutron stars (Haensel et al., 2007).

\section{Compressible Liquid Drop Model}

\section{Liquid Drop Model}

One of models that explains the binding energy is liquid drop model. This model was developed by Neils Bohr with the assumption that the properties of atomic nucleus are similar to properties in liquid drop. These characteristics include (Meyerhof, 1967 ; Arya, 1966) :

a. Its density is constant for all region and does not depend on its size. The size is proportional to the number of particles or molecules in the liquid.

b. The binding energy is proportional to the mass or number of droplet's former.

c. It is homogeneous and cannot be compressed.

d. Evaporation of liquid drops is analogous to the nuclei decay.

By analogizing the nucleus as a liquid drop, so it can be written a semi-empirical mass formula for each atom which has mass $M(A, Z)$. The characteristic of force that arises from nucleons in the nucleus is shortrange and saturation, it is similar to force that apparent from molecules of a liquid. That analogy is the most important prediction in formulating the formula for nucleus mass. First step to construct the mass formula for nucleus is write down the truly nuclei mass, then add corrections that may exist. These corrections include volume, surface, Coulomb, symmetry and oddeven effects and other possible effects. The final result is known as Weizsäcker's semi-empirical mass formula. It will be explained about those corrections.

\section{a. Volume Effect}

Based on liquid drop model, the density of each nucleon is same for all region, so binding energy is proportional to the number of nucleons $(B \sim A)$. Each nucleon is assumed give the same magnitude to bond energy, so the binding energy is $a_{v} A$, where $a_{v}$, is 
volume constant whose value can be obtained from experiment. Thus, bonding energy is equal to volume energy of nucleus, that is

$$
B(A, Z)=a_{v} A
$$

However, because the nucleus is discussed in liquid drop model, there are other effects which causes the magnitude of binding energy such as surface tension and Coulomb force. These effects cause the binding energy in equation (1) should be corrected.

\section{b. Surface Effect}

If nuclei is analogized like liquid drop model, there should be surface tension that occurs in liquid drop. Nucleons that so far in nuclei are pulled from many sides by its nearby nucleons (nucleon in the nucleus and on the surface), while the nucleons on the surface are only attracted from one side (pulled by nucleon in inner nucleus). This causes the binding energy values on the surface to be smaller. This effect has a bigger effect for nucleus which has small $A$ (lighter nuclei) because the distribution/fraction of larger nucleons on the surface compared to the nucleus which has large $A$. Because of this correction, the binding energy in equation (1) must be reduced by the surface energy to be

$$
B=a_{v} A-a_{s} A^{2 / a}
$$

\section{c. Coulomb Effect}

A decrease of binding energy per nucleon in a heavy nuclei (large $A$ ) can be explained by the existence of Coulomb effect. According to Coulomb's law, protons in the nucleus will repeal each other, thereby reducing binding energy or in other words increasing the mass of nucleus. Because the Coulomb force is long-range, each proton affects other proton, not only proton that are near it. Thus, the repulsion force will be stronger by increasing the value of $Z$, with increasing of value $A$. This Coulomb repulsive force causes the average binding energy per nucleon decrease according to increase in $A$.

Therefore the binding energy in equation (2) must be reduced by Coulomb energy. Thus, binding energy can be written as

$$
B=a_{v} A-a_{s} A^{2 / 3}-a_{c} \frac{z(z-1)}{A^{1 / 3}}
$$

\section{d. Symmetry Effect (Nucleon Pair)}

This effect depends on number of proton and neutron in nucleus. Neutron and proton are fermions. Based on Pauli Principle, identical fermions cannot be in the same quantum state. Two same nucleons (proton or neutron) can't occupy a same quantum state. In contrast, proton-neutron can occupy the same quantum state. As the result, proton-neutron pair system has a minimum energy lower than the minimum energy of proton -proton or neutron-neutron system. Lower energy means that bond is stronger, the binding energy is greater. A nuclei that consists of number of proton equal to number of neutron will has a lower minimum energy, which means the binding energy is higher, the bond is more stable, compared to the nucleus with the number of proton are unbalanced with number of neutron.

Thus, the correction for this effect is

$E_{a}=a_{a}\left(\frac{A}{2}-Z\right)^{2} / A$

This correction gives an effect on binding energy, so binding energy in equation (3) must be reduced by the energy $E_{a}$ and becomes

$B=a_{v} A-a_{g} A^{2 / a}-a_{c} \frac{z(Z-1)}{A^{1 / 3}}-a_{p}\left(\frac{A}{2}-Z\right)^{2} / A$

\section{e. Even-Odd Effects}

Another important factor that can influence the binding energy of nuclei is the number of proton and neutron whether odd or even. The most stable nucleus is even even type that has even number of proton and neutron. While the most unstable nuclei is the odd-odd type, that is both proton and neutron are odd number, which means that between proton and neutron will have no pair. The stability of odd-even and even-odd type is almost identical and is in even-even and odd-odd type stability levels. This tendency can be shown by classifying stable nuclei.

By observing these odd-even effects, binding energy needs to be corrected by adding an odd-even pair number $\delta(\mathrm{A}, \mathrm{Z})$ to proton and neutron. This correction is defined by:

$\delta(A, Z)=\left\{\begin{array}{cc}-0.036 \mathrm{~A}^{-\frac{4}{3}} & \text { even } \mathrm{A}, \text { even } \mathrm{Z} \\ 0 & \text { odd } \mathrm{A} \\ 0.036 \mathrm{~A}^{-4 / 3} & \text { even } \mathrm{A} \text {, odd } \mathrm{Z}\end{array}\right.$

So the equation of binding energy after subtracting $\delta$ term becomes

$\mathrm{B}=a_{V} A-a_{g} A^{2 / a}-a_{c} \frac{z(Z-1)}{A^{1 / 3}}-\frac{a_{a}\left(\frac{A}{2}-z\right)^{2}}{A}-\delta(A, Z)$

\section{Compressible Liquid Drop Model}

The developed model from Weizsäcker-Bethe mass formula by adding coefficients of volume, surface and symmetry effects as a density functions are known as compressible liquid drop model. The first model for neutron star crust based on Weizsäcker semi-empirical mass formula, but the formula cannot be used for cluster with very large neutrons in the crust of neutron stars, with Z/A varying from 0.3 to 0.1 (Sulistyani, 2015).

In addition, the presence of neutron fluid influences nuclei cluster in the inner crust. First, neutron liquid reduces the surface energy of nuclei cluster. Second, neutron fluid give pressure on nuclei cluster. A major breakthrough was reached by Baym, Bethe and Pethick (BBP) who applied a compressible liquid-drop model, which included the results of microscopic many-body calculations, to describe consistently both the nucleons in the clusters and the "free" neutrons. BBP illustrates that the energy contribution to liquid drop equation depends not only on $A$ and $Z$, but on a few additional 
parameters, such as, for instance, the size of the cluster and the density of the neutrons and protons inside it.

Because the nucleus is composed of nucleons, interactions between nucleons can affect on nuclei density. One effect that is easily observed is the influence of surface tension. The interaction between nucleons on surface is different from nucleons in the inner (middle) core. Therefore, a review of surface effects is important for nucleon density. When the nucleon density increases, the pressure $P$ will increase. It will affect the equilibrium of density from compressed liquid nucleon. To emphasize this effect, compressible liquid drop model which contains SemiEmpirical Mass Formula that depends on nuclei density is used as the barion density and excess neutron on the inside of nucleus.

Matter at densities below neutron drip $\rho_{N D}$ is not only relevant for neutron star crusts but also for white dwarfs. Following the classical paper of Baym, Pethick and Sutherland, the total energy density in a given layer can be written as (Haensel et al., 2007).

$$
\varepsilon_{\text {tot }}=n_{N} E(A, Z)+\varepsilon_{e}+\varepsilon_{L}
$$

where $n_{\mathrm{N}}$ is the number density of nuclei, $E(A, Z)$ is the energy of a nucleus with $\mathrm{Z}$ protons and $\mathrm{A}-\mathrm{Z}$ neutrons, $\varepsilon_{e}$ is the electron kinetic energy density and $\varepsilon_{L}$ is the lattice energy density, which accounts for the electron-electron, electron-ion and ion-ion Coulomb interactions.

The total energy of cluster needs to be renewed for inner crust of neutron star, because of contribution from neutron gas. Then equation (8) in liquid drop model, the total energy density can be written by adding the energy of neutron gas $\varepsilon_{n}$ (Chamel and Haensel, 2008) i.e.

$$
\varepsilon_{\text {tot }}=n_{N} E(A, Z)+\varepsilon_{e}+\varepsilon_{L}+\varepsilon_{n}
$$

The nuclear clusters are treated as liquid drops of nuclear matter whose energy can be decomposed into volume, surface and coulomb terms.

$$
E\left\{A_{,} Z\right\}=E_{N_{i} v o l}+E_{N_{s} \text { surf }}+E_{N_{i} \text { coul }}
$$

where $E_{N_{j} \text { vol }}, E_{N_{s} \text { surf }}, E_{N_{j} \text { coul }}$ are volume energy, surface energy, and Coulomb energy, respectively. Pair and skin term are ignored because only macroscopic properties of ground state are reviewed. In the simplest version, the drop is supposed to be incompressible with a density on the order of $\rho_{0}$ corresponding to the density inside heavy nuclei. This implies that the volume and surface terms in Equation (10) are proportional to $\mathrm{A}$ and $\mathrm{A}^{2 / 3}$, respectively (Chamel and Haensel, 2008). Each contribution to the energy, Equation (10), can then be parameterized in terms of the numbers $\mathrm{A}$ and $\mathrm{Z}$. The volume contribution in equation (10) is given by

$$
E_{N_{u} \text { Vol }}=\varepsilon\left\{n_{\text {ni }}, n_{\text {pi }}\right\} V_{N}
$$

where $\varepsilon\left\{n_{m i}, n_{p i}\right\}$ is the energy density of homogeneous nuclear matter and $n_{n i}, n_{p i}$ are respectively the neutron and proton densities inside the clusters. $V_{N}$ is the volume of the cluster. For consistency the energy density of the surrounding neutron gas is expressed in terms of the same function $\varepsilon\left\{n_{\text {mi }}, n_{\text {pi }}\right\}$ as

$\varepsilon_{n}=\varepsilon\left\{n_{n o}, 0\right\}(1-w)$

where $n_{n o}$ is the number density of free neutrons outside the clusters and $w$ is the volume fraction of the cluster.

$w=\frac{V_{N}}{V_{\text {CEII }}}=\left(\frac{r_{p}}{R_{\text {cell }}}\right)^{3}$

The surface thermodynamic potential per unit area $\sigma$ and the chemical potential $\mu_{m s}$ of neutrons adsorbed on the surface of the drop (forming a neutron skin) by

$\sigma=\left.\frac{\partial \mathrm{E}_{\text {N surf }}}{\partial \mathrm{A}}\right|_{N s}$

$\mu_{n s}=\left.\frac{\partial \mathrm{E}_{N_{\text {surf }}}}{\partial N_{S}}\right|_{A}$

where $A$ is the surface area of the cluster and $N_{s}$ is the number of adsorbed neutrons. Since energy is an extensive thermodynamic variable, it follows from Euler's theorem about homogeneous functions that (Chamel and Haensel., 2008)

$\mathrm{E}_{N, \text { surf }}=\sigma \mathrm{A}+N_{s} \mu_{n s}$

The magnitude Coulomb energy from a uniformlycharged spherical drop of radius $r_{p}$ is given by

$E_{N_{i} \text { Coul }}=\frac{3}{5} \frac{Z^{2} e^{2}}{r_{p}}$

Compressible liquid drop model can be used also to determine the energy of the Wigner-Seitz cell, where $E_{s e l}$ is the total energy of nuclear cluster energy, the neutron gas contribution and the electron energy $E_{e}$ given by (Sulistyani, 2015).

$E_{\text {sel }}=E_{N, \text { bulk }}+E_{N, \text { surf }}+E_{N_{i} \text { coul }}+E_{\text {a }}$

where $E_{N, \text { butk }}$ is the volume energy for cells in which there is a contribution of neutron gas. For the magnitude $E_{N_{s} \text { bulk }}$ is given by

$E_{N_{3} \text { bulk }}=V_{\text {cell }}\left[w \varepsilon_{N_{2}, i}+(1-w) \varepsilon_{n}\right]$

where $\varepsilon_{N, i}=\varepsilon\left\{n_{m i}, n_{p i}\right\}$ is nuclear energy density and $\varepsilon_{n}$ is the energy density of neutron gas. In the Wigner-Seitz approximation the magnitude of surface energy is affected by surface tension factor $\sigma_{s}$ and thickness of neutron skin $s_{n}$. This can be seen from the following equation

$E_{N_{s} \text { surf }}=\left(\sigma_{s}+s_{n}\left(n_{n i}-n_{n o}\right) \mu_{n s}\right) A$

The thickness of neutron skin $s_{n}$ is defined as (Douchin and Haensel., 2000).

$s_{n}=r_{n}-r_{p}$

Where $r_{n}$ and $r_{p}$ are the neutron radius and the proton radius, respectively. When crytal lattice was in 
mechanical equilibrium, we will find.

$$
E_{N_{\text {surf }}}=2 E_{\text {coul }}
$$

where $E_{\text {cour }}$ the total Coulomb energy whose magnitude depends on lattice energy. So the total Coulomb energy is the sum of contribution from nucleus and lattice, which is mathematically formulated as

$$
E_{\text {couI }}=E_{N_{2} \text { Coul }}+V_{\text {sel }} \varepsilon_{\text {L }}
$$

According to Wigner-Seitz approximation, the magnitude of lattice energy is (Shapiro and Teukolsky, 1983)

$$
\varepsilon_{L}=-\frac{9}{10}\left(\frac{4 \pi}{3}\right)^{\frac{1}{3}} Z^{\frac{2}{s}} e^{2} n_{e}^{\frac{4}{3}}
$$

by combining the equation $E_{N_{\alpha} \text { Cour }}$ and $\varepsilon_{L}$, the equation for coulomb energy is found in total.

$E_{\text {coul }}=E_{N_{1} \text { Coul }} f_{3}(w)$

where $f_{3}(w)$ is dimensionless function and given by

$$
f_{3}(w)=1-\frac{3}{2} w^{\frac{1}{s}}+\frac{1}{2} w
$$

The equation for electron energy is (Roca-Maza and Piekarewicz, 2008 ; Sharma et al., 2015)

$$
\varepsilon_{B}=\frac{m_{\varepsilon}^{4}}{8 \pi^{2} m_{b}}\left(x_{F} y_{F}\left(x_{F}^{2}+y_{F}^{2}\right)-\ln \left(x_{F}+y_{F}\right)\right)
$$

where parameter $x_{F}$ and $y_{F}$ defined as

$$
\begin{aligned}
& x_{F}=\frac{p_{F_{B}}}{m_{e} c} \\
& y_{F}=\frac{E_{P_{e}}}{m_{e}}=\sqrt{1+x_{F}^{2}}
\end{aligned}
$$

\section{Neutron Stars}

\section{The Structure of Neutron Stars}

Generally, the region of neutron star is divided into core and sheath. The core of star consists of the outer core and inner core. And sheath consists of a dense crust with atomic nucleus arranged into crystals, and a ocean composed of Coloumb Fluid. Structure of star sheath consists of atmosphere, ocean, outer crust, inner crust, and mantle as shown in figure (1).

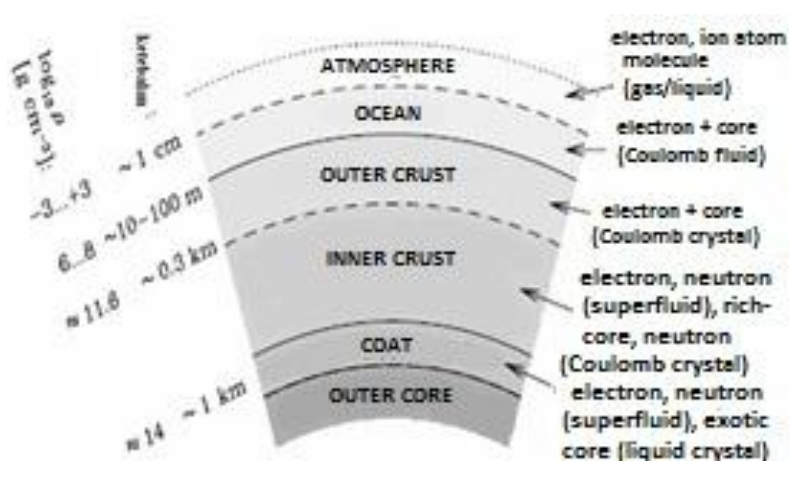

Figure 1. The structure of sheath of neutron stars (Sulistyani, 2010).

\section{a. Atmosphere}

The atmosphere is a thin plasma layer, where the spectrum of thermal electromagnetic neutron star radiation is formed. The spectrum, beaming and polarization of emerging radiation can be determined theoretically by solving the radiation transfer problem in atmospheric layers. This radiation contains valuable information on the parameters of the surface layer (on the effective surface temperature, surface gravity, chemical composition, strength and geometry of the surface magnetic field) and on the masses and radii of neutron stars. The atmosphere thickness varies from some ten centimeters in a hot neutron star (with the effective surface temperature $T_{s} \sim 3 \times 10^{6} \mathrm{~K}$ ) to a few millimeters in a cold one $\left(T_{s} \sim 3 \times 10^{5} \mathrm{~K}\right)$. Very cold or ultramagnetized neutron stars may have a solid or liquid surface. If the radiation flux is too strong, the radiative force exceeds the gravitational one and makes the atmosphere unstable with respect to a plasma outflow. In a hot nonmagnetized atmosphere, where the radiative force is produced by Thomson scattering, this happens whenever the stellar luminosity $L$ exceeds the Eddington limit (Haensel et al, 2007).

\section{b. Ocean}

Depth of ocean of neutron stars $~ 10-100 \mathrm{~m}$, with mass density $\rho \sim 10^{6}-10^{9} \mathrm{~g} \mathrm{~cm}^{-3}$. The ocean contains of a neutron by degenerated electrons. This region contains of neutron superfluides that smaller than superfluid of proton and electron. The ocean is unstable due to influence of temperature and chemical composition on the surface of neutron stars.

\section{c. Outer Crust}

Its matter consists of ions $Z$ and electrons $e$. A very thin surface layer (up to few meters in a hot star) contains a non-degenerate electron gas. In deeper layers the electrons constitute a strongly degenerate, almost ideal gas, which becomes ultrarelativistic at $\rho \gg 10^{6} \mathrm{gr} \mathrm{cm}^{-3}$. The total pressure is determined by degenerated electrons. In the outer atmosphere layers the ions may constitute a Boltzmann gas, but in deeper layers they form a strongly coupled Coulomb system (liquid or solid). This induces beta captures in atomic nuclei and enriches the nuclei with neutrons. At the base of the outer crust the neutrons start to drip out from the nuclei producing a free neutron gas (Haensel et al., 2007).

\section{d. Inner Crust}

The inner crust may be about one kilometer thick. The density $\rho$ in the inner crust varies from $\rho_{\text {ND }}$ at the upper boundary to $\sim 0.5 \rho_{0}$ at the base (Chamel and Haensel, 2008). The matter of the inner crust consists of electrons, free neutrons $n$, and neutron-rich atomic nuclei. The fraction of free neutrons increases with growing $\rho$. The neutronization at $\rho \approx \rho_{N D}$ greatly softens the EOS, but at the crust bottom the repulsive short-range component of the neutron-neutron 
interaction comes into play and introduces a considerable stiffness. In the bottom layers of the crust, in the density range from $\frac{1}{3} \rho_{0}$ to $\frac{1}{2} \rho_{0}$ the nuclei may become essentially nonspherical and form a "mantle" (Haensel et al., 2007).

\section{e. Mantle}

The mantle is a layer on a neutron star that separates the star's core from inner crust. The structure of star's core is divided into the outer and inner core, with mass filled $99 \%$ of the total mass of neutron star.

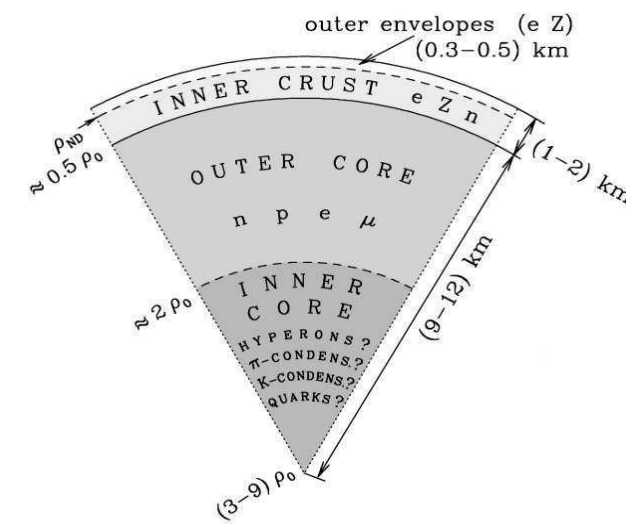

Figure 2. The structure of neutron star's core (Haensel et al., 2007).

\section{f. Outer Core}

It is several kilometers thick. Its matter consists of neutrons with several percent admixture of protons $\mathrm{p}$, electrons, and possibly muons $\mu$ (the so called npe $\mu$ composition). The state of this matter is determined by the conditions of electric neutrality and beta equilibrium, supplemented by a microscopic model of many-body nucleon interaction. The beta equilibrium implies the equilibrium with respect to the beta (muon) decay of neutrons and inverse processes. All npe $\mu$ plasma components are strongly degenerate. The electrons and muons form almost ideal Fermi gases. The neutrons and protons, which interact via nuclear forces, constitute a strongly interacting Fermi liquid and can be in superfluid state.

\section{g. Inner Core}

The inner core, where $\rho \geqslant 2 \rho_{0}$, occupies the central regions of massive neutron stars (and does not occur in low-mass stars whose outer core extends to the very center). Its radius can reach several kilometers, and its central density can be as high as $(10-15) \rho_{0}$. Its composition and the EOS are very model dependent. Some of the hypotheses are material hyperionization, pion condensation, kaon condensation, and the transition phase of the oceanic layer to quark material (Haensel et al, 2007).

\section{The Ground State of Inner Crust of Neutron Stars}

The inner crust of neutron stars is about one kilometers thick. It has varying density in some region. The density in the upper layer is $\rho \approx 4.32 \times 10^{11} \mathrm{gr} \mathrm{cm}^{-3}$. Meanwhile the density in the base is about $0,5 \rho_{o}$, where $\rho_{o}$ is density of normal nuclear.
With increasing density, the ground-state value of Z/A decreases and neutrons become less and less bound. A bonding in neutrons that decrease affects neutron drip phenomena, that is $\rho \sim \rho_{N D}$. Neutron drip phenomena related with chemical potential of pure neutron $\mu_{n}{ }^{\prime}$. As a simple, chemical potential is a tendency of component exit from its parent or in the other definition, the motion of particle from one phase to the other phase.

As long as $\mu_{n}{ }^{\prime}<0$, all neutrons are bound within nuclei. The neutron drip point corresponds to $\mu_{n}{ }^{\prime}=0$, beyond this point neutrons "drip out of nuclei", i.e. they begin to fill states in the continuous part of the energy spectrum.

Chemical potential of a neutron in a nucleus is defined by (Chamel and Haensel, 2008).

$\mu_{m}{ }^{\prime}=\mu_{n}-m_{m} c^{2}=\left(\frac{\partial E[A, Z]}{\partial N}\right)_{z}-m_{n} c^{2}$

Where $\mu_{m}$ is chemical potential of neutron and $m_{n} c^{2}$ is rest-mass energy of neutron. We can roughly localize the neutron drip point using the approximate mass formula for $E^{\prime}\{A, Z\}=E\{A, Z\}-A m c^{2}$, where, for simplicity, we neglect the neutron-proton mass difference, putting $m_{n} \approx m_{p} \approx m=939 \mathrm{MeV} / c^{2}$ and $A m c^{2}$ is total of rest mass energy nucleon. Neglecting surface and Coulomb terms, we have (Chamel and Haensel, 2008)

$E^{\prime}\{A, Z\}=A\left(E_{v o l}+E_{s y m} \delta^{2}\right)$

Where $\delta \equiv(\mathrm{N}-\mathrm{Z}) / \mathrm{A}$, and $E_{\text {vol }}$ and $E_{\text {sym }}$ are nuclear volume and symmetry energies, respectively. Experimentally, $E_{\text {vol }} \cong-16 \mathrm{MeV}$ and $E_{s y m}$ has magnitude $32 \mathrm{MeV}$ (Chamel and Haensel, 2008).

The inner crust of a neutron star is a unique system, which is not accessible in the laboratory due to the presence of this neutron gas. In the following we shall thus refer to the "nuclei" in the inner crust as "clusters" in order to emphasize these peculiarities. The description of the crust beyond neutron drip therefore relies on theoretical models only. The presence of neutron gas affect matter in inner crust to be compressible as the result of pressure from neutron gas. The inner crust of neutron stars is assumed like lattice in the Wigner-Seitz approximation as can be seen in fig [3], which has cubic structure or more complex. But the lattice can be substituted by spheres cell in the same of volum.

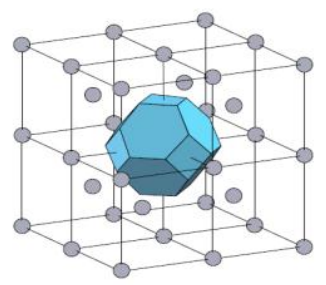

Figure 3. The structure of body centered cubic in Wigner-Seitz approximation (Chamel and Haensel, 2008). 
In the Wigner-Seitz approximation the crystal (represented here as a two-dimensional hexagonal lattice) is decomposed into independent identical spheres, centered around each site of the lattice. The radius of the sphere is chosen so that the volume of the sphere is equal to $1 / n_{N}$, where $n_{N}$ is the density of lattice sites (ions) as in Fig. System is considered as cell unit composed of one nuclei, where the density is given by $n_{N}=n_{b} / A$ and the volum is $V_{c}=1 / n_{N}$, radii of cell unit is equal to $r_{c}=\left(\frac{4 \pi n_{N}}{3}\right)^{-1 / 3}$ (where $r_{c}$ is called as ion's radius).

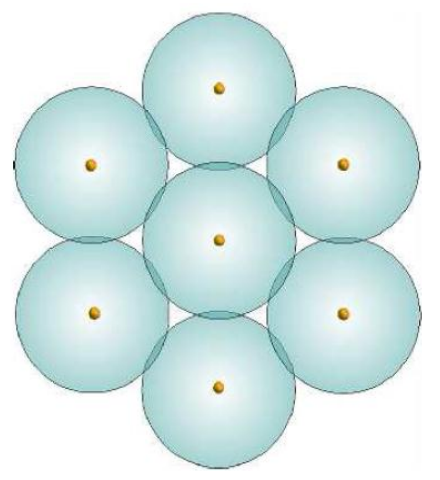

Figure 4. In the Wigner-Seitz approximation the crystal (represented here as a two-dimensional hexagonal lattice (Chamel and Haensel, 2008).

\section{The Ground State of Pasta of Neutron Stars}

At the base of the inner crust we find neutron rich nuclei with a free neutron gas. The proton fraction here is near $5 \%$. At densities above $0.2 n_{0}$, the nuclei begin to touch and fuse forming complex shapes. As the density approaches $n_{0}$, the complex shapes transition to uniform nuclear matter. These shapes have since come to be called nuclear pasta, due to their resemblance to spaghetti and lasagna and other namesake pasta. In total, for a $10 \mathrm{~km}$ radius NS, the ions of the crust may extend to a depth of about $1 \mathrm{~km}$, and the pasta region may extend an additional $100 \mathrm{~m}$ (Caplan and Horowitz, 2017).

In the densest layers of the crust the Coulomb energy is comparable in magnitude to the net nuclear binding energy. The matter thus becomes frustrated and can arrange itself into various exotic configurations as observed in complex fluids. Ogasawara \& Sato suggest that as the nuclei fill more and more space, they will eventually deform, touch and merge to form new structures. Baym, Bethe and Pethick predicted that as the volume fraction exceeds $1 / 2$, the crust will be formed of neutron bubbles in nuclear matter. In the general framework of the compressible liquid drop model considering the simplest geometries, Hashimoto and friends show that as the nuclear volume fraction $\mathrm{w}$ increases, the stable nuclear shape changes from sphere to cylinder, slab, tube and bubble, as illustrated in Figure [4] .

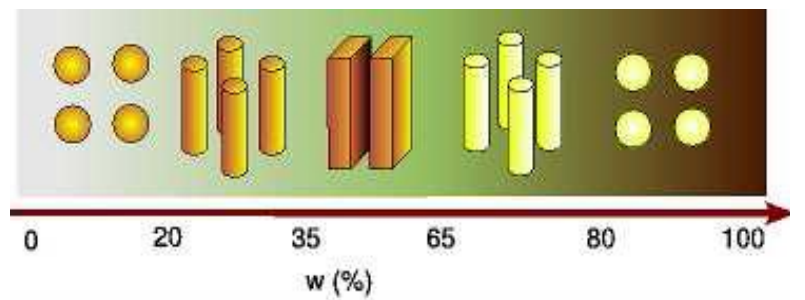

Figure 5. Sketch of the sequence of pasta phases in the bottom layers of ground-state crusts with an increasing nuclear volume fraction (Chamel and Haensel, 2008)

The shape of nuclei can be separated corresponding to dimensionality. Dimensionality has a value from one till three. For $d=3$ related with spherical nuclei in a nucleon gas and spherical bubbles in a denser nuclear matter. For $d=2$, the nuclear structures were cylindrical nuclei and cylindrical holes in the nuclear matter filled with the nucleon gas. And finally for $\mathrm{d}=1$ it's slab structure (Haensel et al., 2007).

According to the Bohr-Wheeler fission condition an isolated spherical nucleus in vacuum is stable with respect to quadrupolar deformations if

$E_{\text {N,Coul }}^{0}<2 E_{\text {N,Surf }}^{0}$

where $E_{N, c o u l}^{0}$ and $E_{N, S u r f}^{0}$ are the Coulomb and surface energies of the nucleus, respectively. The superscript (0) reminds that a nucleus in vacuum. It is found that spherical clusters become unstable to quadrupolar deformation if $w>w_{\text {hritis }}=1 / 8$ (Chamel and Haensel, 2008). In pasta of neutron stars, relation of fraction of volume occupied by the clusters $\mathrm{w}$ with dimensionality is

$w=\left(\frac{r_{p}}{r_{0}}\right)^{d}$

where $r_{p}$ is nucleus radii and $r_{c}$ is Wigner-Seitz cell radius (Haensel et al., 2007). Every shape of nuclei in pasta will get the deformation. The deformation means displacement of nucleus from the first position into any position with polar angle $\theta$. Let us considered a nucleus as a spherical liquid drop of radius $\mathrm{R}$ and total charge $Z e$ in which neutrons and protons are distributed uniformly, the distance from the center of the drop to an arbitrary point on the surface with polar angle:

$X(\theta)=R\left(1+\alpha_{0}+\alpha_{2} P_{2}(\cos \theta)+\cdots\right)$

Relation of $\alpha_{0}$ and $\alpha_{2}$ be expressed as (Iida et al., 2001)

$\hat{a}_{0}=-\left(\frac{1}{5}\right) \alpha_{2}^{2}$

Next, derive a fission-instability condition appropriate for a nuclear rod, composed of uniformly distributed neutrons and protons (proton charge density $\rho)$ as well as having a circular section (sectional radius $R$ ) and an infinitely long axis. For rod, a small sectional deformation of the quadrupole type that is uniform in the direction of the axis. This deformation is characterized by the distance, measured on a horizontal 
section, from the axis to a point located on the surface with a given angle $\theta$ :

$$
X(\theta)=R\left(1+\hat{a}_{0}+\alpha_{2} \cos 2 \theta+\cdots\right)
$$

Relation of $\alpha_{0}$ and $\alpha_{2}$ be expressed as (Iida et al., 2001)

$$
\alpha_{0}=-\left(\frac{1}{4}\right) \alpha_{2}^{2}
$$

As the density of the matter increases, the distance between neighboring nuclei decreases. Hashimoto and friends assumed that the constituents of the matter are electrons, protons and neutrons, of which the electrons are distributed over the whole space and the protons are confined in nuclei. Neutrons may be either distributed over the whole space or confined in nuclei (Hashimoto et al., 1984). To know more detail, this following will be explained shape of nucleus.

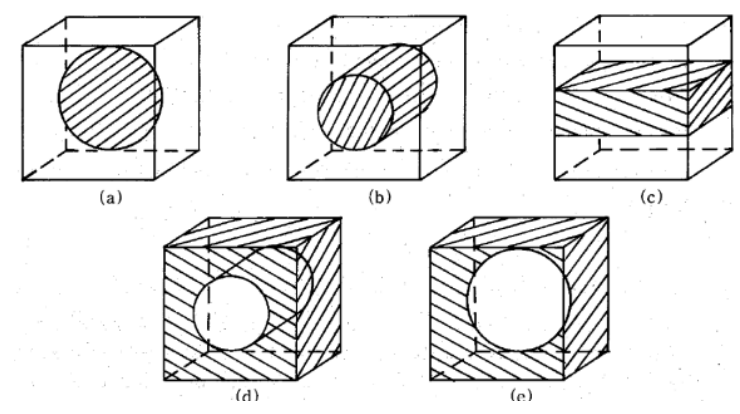

Figure 6. Candidates for nuclear shapes. Protons are confined in the hatched regions, which we call nuclei. Then the shapes are, (a) Sphere, (b) Cylinder, (c) Board or plank, (d) Cylindrical hole and, (e) Spherical hole (Hashimoto et al., 1984).

An example of a soft astromaterial is "nuclear pasta," found in the inner crust of neutron stars. Under compression, the ions in the crust will rearrange into exotic shapes in order to minimize their energy. The competition between the nuclear attraction of protons and neutrons and the Coulomb repulsion between protons creates a variety of nonspherical nuclei. This transition is now believed to involve several pasta phases.

One of model to analyze the structure of pasta is semi-classical molecular dynamics (Caplan and Horowitz, 2017). This model treats protons and neutrons as point particles, and uses a set of three classical two-body potentials for describing their interaction:

$$
\begin{aligned}
& V_{n p}(r)=a e^{-\frac{r^{2}}{M}}+[b-c] e^{-\frac{r^{2}}{2 M}}, \\
& V_{n n}(r)=a e^{-\frac{r^{2}}{M}}+[b+c] e^{-\frac{r^{2}}{2 M}} \\
& V_{p p}(r)=a e^{-\frac{r^{2}}{M}}+[b+c] e^{-\frac{r^{2}}{2 M}}+\frac{\alpha}{r} e^{-\frac{r}{A}}
\end{aligned}
$$

Where the subscripts $n$ and $p$ denote the interactions between neutrons and protons and $r$ is the inter-particle separation. The nucleons interact by a short range potential meant to model the nuclear interaction whose strength and range are determined by the parameters $a, b, c$ and $\Lambda$, which are given in Table 1 .

Table 1. Parameters of the nuclear interaction (Caplan and Horowitz, 2017).

\begin{tabular}{llll}
\hline $\boldsymbol{a}(\mathrm{MeV})$ & $\boldsymbol{b}(\mathrm{MeV})$ & $\boldsymbol{c}(\mathrm{MeV})$ & $\boldsymbol{A}\left(\mathrm{fm}^{2}\right)$ \\
\hline $\mathbf{1 1 0}$ & -26 & 24 & 1,25 \\
\hline
\end{tabular}

The parameter $a$ defines the strength of the shortrange repulsion between nucleons, $b$ and $c$ the strength of their intermediate-range attraction and $\Lambda$ the length scale of the nuclear potential (Schneider et al., 2013). According to Thomas-Fermi approximation, total energy of an ensemble of $A-Z$ neutrons, $Z$ protons, and $Z$ electrons in a spherical Wigner-Seitz (WS) cell of volume $V_{c}=\frac{4 \pi\left(R_{c}\right)^{3}}{3}$ can be expressed as (Sharma et al., 2015)

$$
\begin{aligned}
& E=E\left(A_{i} Z, R_{c}\right)=\int d V\left[H\left(n_{n}, n_{p}\right)+m_{n} n_{n}+\right. \\
& m_{p} n_{p}+\varepsilon_{e l}\left(n_{\theta}\right)+\varepsilon_{\text {coul }}\left(n_{p}, n_{\varepsilon}\right)+\varepsilon_{\text {ex }}\left(n_{p}, n_{\theta}\right)
\end{aligned}
$$

Where $d V$ is volume element of Wigner-Seitz cell, $H\left(n_{n}, n_{p}\right)$ is the magnitude of nuclei energy density, and $\varepsilon_{e x}\left(n_{p}, n_{e}\right)$ is exchange energy. The magnitude of $H\left(n_{n}, n_{p}\right)$ is (Viñas et al., 2017)

$H\left(n_{n}, n_{p}\right)=\frac{a}{5}\left(3 \pi^{2}\right)^{2 / a}\left[\frac{\hbar^{2}}{2 m_{n}} n_{n}^{\frac{5}{3}}+\frac{\hbar^{2}}{2 m_{p}} n_{p}^{\frac{5}{3}}\right]+$ $V\left(n_{n}, n_{p}\right)$

The term $\varepsilon_{e x}\left(n_{p}, n_{e}\right)$ in Eq. (40) is the exchange part of the proton-proton and electron-electron interactions treated in Slater approximation (Sharma et al., 2015)

$\varepsilon_{e x}\left(n_{p}, n_{\varepsilon}\right)=-\frac{3}{4}\left(\frac{3}{\pi}\right)^{\frac{1}{3}} e^{2}\left(n_{p}^{\frac{4}{3}}+n_{n}^{\frac{4}{3}}\right)$

By assuming that the electrons are uniformly distributed, $\varepsilon_{\text {coul }}\left(n_{p}, n_{e}\right)$ term can be written as (Viñas et al., 2017).

$$
\varepsilon_{\text {coul }}\left(n_{p}, n_{\varepsilon}\right)=\frac{1}{2}\left(n_{p}(r)-n_{\varepsilon}\right)\left(V_{p}(r)-V_{\theta}(r)\right)
$$

Where equation for $V_{p}(r)$ and $V_{e}(r)$ are given by

$$
\begin{aligned}
& V_{p}(r)=\int \frac{e^{\mathrm{z}} n_{p}\left(r^{z}\right)}{\| r-r^{\prime} \mid} d r^{\prime} \\
& V_{e}(r)=\int \frac{e^{\mathrm{a}} n_{e}}{\left\|r-r^{7}\right\|} d r^{y}
\end{aligned}
$$

\section{Symmetry Energy}

The role of symmetry energy is essential to understand the mechanism of stability of very-neutron rich nuclei, but it is also related to many phenomena occurring in neutron stars. The stability of matter inside neutron stars is very sensitive to $E_{s y m}$ and its first derivative. 
Around saturation density, neutrons tend to decay to protons through $\beta$-decay, and the cooling of neutron stars is strongly connected to the proton/neutron ratio as a function of density. This ratio is mainly governed by behavior of $E_{s y m}$ as a function of density (Gandolfi, 2012). For baryonic matter composed solely of neutrons and protons, proton fraction can be expressed as (Lattimer and Steiner, 2014)

$x=\frac{n_{p}}{n_{n}+n_{p}}$

Nucleus that composed of number of protons equal to number of neutrons is known as symmetric nuclear matter (SNM). Meanwhile for matter that composed of neutrons only called as pure neutron matter (PNM). The symmetry energy $E_{s y m}(\rho)$ is defined as the difference between the energy per baryon of pure neutron matter and the energy per baryon of infinite homogeneous nuclear matter with equal neutron number density, $\rho_{n}$, and proton number density, $\rho_{p}$. Symmetry energy is formulated as (Gandolfi et al., 2013).

$$
E_{\text {sym }}(\rho)=E(\rho, 0)-E_{0}(\rho)
$$

Where $E(\rho, 0), E_{0}(\rho)$ is EoS of pure neutron matter and symmetryc nuclear matter, respectively. The energy per baryon of isospin asymmetric nuclear matter can be expanded in even powers of x (Gandolfi et al., 2013),

$$
E(\rho, x)=E_{0}(\rho)+E_{s y m}^{(2)}(1-2 x)^{2}+E_{s y m}^{(4)}(1-2 x)^{4}+\cdots
$$

The equation for symmetry energy around the saturation density can be expressed as (Vida $\tilde{n} a$ dan Providência, 2010).

$$
\begin{aligned}
& E_{s y m}(\rho)=E_{s y m}+\frac{L}{3} \frac{\rho-\rho_{0}}{\rho_{0}}+\frac{K_{s y m}}{2}\left(\frac{\rho-\rho_{0}}{3 \rho_{0}}\right)^{2}+ \\
& \frac{Q_{s y m}}{6}\left(\frac{\rho-\rho_{0}}{3 \rho_{0}}\right)^{3}+O(4)
\end{aligned}
$$

Where $E_{\text {sym }}$ is symmetry energy when saturation (generally written in $\mathrm{S}$ ) and L corresponds to slope of $\mathrm{E}_{\mathrm{sym}}(\mathrm{p}) \times \mathrm{L}, \mathrm{K}_{\mathrm{sym}}$, and $\mathrm{Q}_{\mathrm{sym}}$ are given by (Providência et al., 2013)

$$
\begin{aligned}
& L=\left.3 \rho_{0} \frac{\partial E_{s y m}}{\partial \rho}\right|_{\rho=\rho_{0}} \\
& K_{s y m}=\left.9 \rho_{0}^{2} \frac{\partial^{\mathrm{z}} E_{s y m}(\rho)}{\partial \rho^{\mathrm{z}}}\right|_{\rho=\rho_{0}} \\
& Q_{s y m}=\left.27 \rho_{0}^{3} \frac{\partial^{\mathrm{s}} E_{s y m}(\rho)}{\partial \rho^{\mathrm{s}}}\right|_{\tilde{\mathrm{n}}=\rho_{0}}
\end{aligned}
$$

For pure neutron matter at $n_{s}$ and in the quadratic approximation, the energy and pressure are given by

$$
\begin{aligned}
& E_{N}\left(n_{s}\right)=E(n, 0) \cong S_{v}+B \\
& P_{N}\left(n_{s}\right)=p\left(n_{s_{s}} 0\right)=n_{s}^{2}\left(\frac{\partial E}{\partial n}\right)_{n_{s} x=0} \approx \frac{L}{3} n_{s}
\end{aligned}
$$

Where $n_{s}=\rho_{0}, \quad S_{v}=E_{s y m}, n=\rho, \quad$ and $B=-E\left(n_{s}, \frac{1}{2}\right)=16 \mathrm{MeV} . B$ is the binding energy of symmetric matter at the saturation density. For matter in $\beta$-equilibrium, it follows that.

$P_{\beta}\left(n_{s}\right) \approx \frac{L}{3} n_{s}\left[1-\left(\frac{4 S_{V}}{\hbar c}\right)^{3} \frac{4-\frac{B S_{V}}{L}}{3 \pi^{2} n_{s}}+\cdots\right]$

The magnitude of energy per particle from symmetric nuclear matter described as

$E\left(n, x=\frac{1}{2}\right)=m_{N} c^{2}+\frac{3}{5} E_{F}^{0} u^{\frac{2}{3}}+V(u)$

Where $m_{N} c^{2}$ is total rest-mass energy of baryon and $E_{F}^{o}$ corresponds to Fermi energy per baryon in equilibrium state. While $V(u)$ potential energy as a function $u . u$ is ratio between density of symmetric nuclear matter and saturation density. Mathematically $u$ written as.

$u=\frac{n}{n_{s}}$

Fermi energy per baryon $E_{F}^{o}$ can be expressed as (Psonis et al., 2008).

$E_{F}^{o}=\frac{\left(h k_{F}^{0}\right)^{2}}{2 m_{N}}$

The density dependent potential energy per nucleon $\mathrm{V}$ (u) of the symmetric nuclear matter is parameterized, as follows (Prakash et al., 1988).

$$
\begin{aligned}
& V(u)=\frac{1}{2} A u+\frac{B u^{\sigma}}{1+B^{\prime} u^{\sigma-1}}+3 \sum_{\mathrm{i}=1,2} C_{\mathrm{i}}\left(\frac{A_{\mathrm{i}}}{p_{\mathrm{p}}^{\mathrm{p}}}\right)^{3}\left(\frac{p_{F}}{A_{i}}-\right. \\
& \left.\arctan \frac{p_{F}}{A_{\mathrm{i}}}\right)
\end{aligned}
$$

Where $p_{F}$ is the Fermi momentum. The parameters $A_{1}$ and $A_{2}$ parameterize the finite forces between nucleons. The parameters $A_{v} B, B^{r}, \sigma_{v} C_{1_{1}}$ and $C_{2}$ are determined with the constraints provided by the properties of nuclear matter saturation.

\section{MATERIALS AND METHODS}

This research is using literature study method. The literature study conducted by the author is to search on various written sources, whether in the form of books, articles, manuscripts and journals, or documents relevant. Combine collected data, analyzed, and make decisions.

\section{RESULTS AND DISCUSSION}

One of the virtues of the CLDM is its flexibility as far as the shape of nuclei is concerned. The terms $\varepsilon_{N, \text { butk }}$ and electron energy are shape independent. The surface and Coulomb terms do depend on the shape, but they can easily be calculated neglecting the curvature corrections. In compressible liquid drop model, electrons are assumed to form a uniform Fermi gas, and 
yield the rest plus kinetic energy contribution, denoted by $E_{e}$.

Basically the differences of shape of nuclei can be determined by dimensionality. So, the equation of surface energy in equation (20) should be modified to be

$\varepsilon_{N, S u r f}=\frac{w d}{r_{p}}\left[\left(n_{n i}-n_{n o}\right) \mu_{n} s_{n}+\sigma_{s}\right]$

Coulomb energy in equation (25) should be modified too because of the dimensionality of nuclei. The equation for Coulomb energy can be written as

$\varepsilon_{\text {Coul }}=\frac{4 \pi}{5}\left(n_{p, i} e r_{p}\right)^{2} f_{d}(w)$

where $f_{a}(w)$ given by

$f_{d}(w)=\frac{5}{d+2}\left[\frac{1}{d-2}\left(1-\frac{1}{2} w^{1-\frac{\pi}{d}} d\right)+\frac{1}{2} w\right]$

In the case of $d=2$ (rods) one has to take the limit of $d$ $\rightarrow 2$ which gives

$f_{2}(w)=\frac{5}{8}\left[\ln \frac{1}{w}-1+w\right]$

These formulae hold also for neutron gas tubes and neutron gas bubbles but in these cases one has to replace $w$ into $1-w$ and $r_{p}$ stands for the radius of tubes or bubbles. The magnitude of pressure from one system that composed of some or many baryons given by

$$
P_{j}^{\text {bulk }}=n_{j}^{2} \frac{\theta}{\partial n_{j}}\left[\frac{a_{\text {bulk }}}{n_{j}}\right]
$$

We will find the equation for the pressure equilibrium condition, that is (Haensel et al., 2007).

$P_{i}^{\text {bulk }}-P_{0}^{\text {bulk }}=$

$(d-1) \frac{\sigma_{s}}{r_{v}}+\frac{4 \pi}{5} e^{2} n_{p, i}^{2} r_{p}^{2} f_{d}(w)\left[\frac{2}{d}+\frac{w f_{d}^{\prime}}{4 f_{d}(w)}-1\right]$

All characteristics in pasta of neutron stars after using compressible liquid drop model are:

\section{The Relation of Energy Per Baryon and Baryon Density}

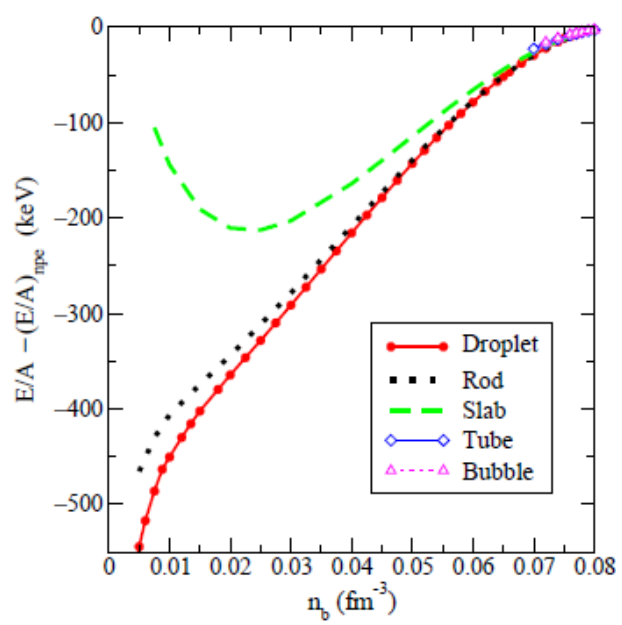

Figure 7. Energy per baryon of different shapes relative to uniform npe matter as a function of baryon density in the inner crust (Sharma et al., 2015).

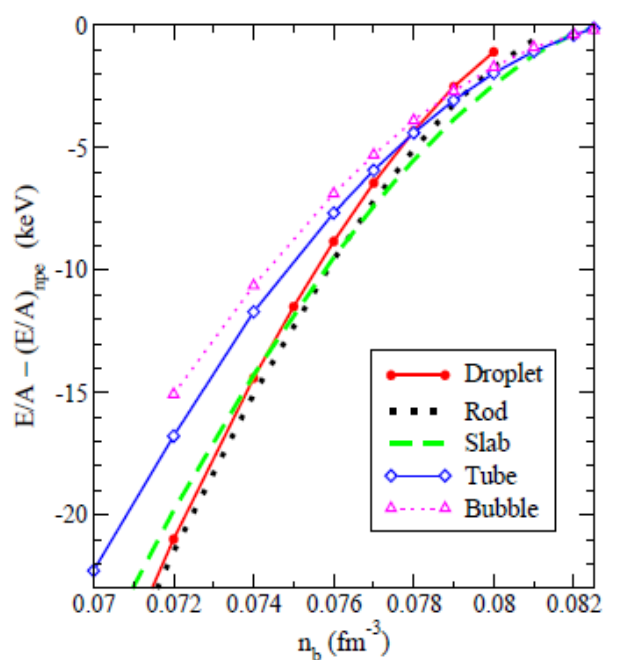

Figure 8. Energy per baryon of different shapes relative to uniform npe matter as a function of baryon density in the high-density region of the inner crust (Sharma et al., 2015).

In Fig [3], the results for the minimal energy per baryon E/A in the different shapes. The energy per baryon is shown relative to the value in uniform neutron-proton-electron (npe) matter in order to be able to appreciate the energy separations between shapes.

It can be seen in Fig [4] that the cylindrical shape is the energetically favoured configuration up to a crustal density of $0.076 \mathrm{fm}^{-3}$ where a second change takes place to the planar slab shape. As the density of the crustal matter increases further, the energy per baryon of tubes and bubbles becomes progressively closer to that of the slabs (Sharma et al., 2015).

The spherical droplet configuration is the energetically most favourable shape all the way up to $n_{b} \sim 0.065 \mathrm{fm}^{-3}$, see Fig. 3 . When the crustal density reaches $\sim 0.065 \mathrm{fm}^{-3}$ (approximately $10^{14} \mathrm{~g} / \mathrm{cm}^{3}$ ), the nucleus occupies a significant fraction of the cell volume and it may happen that non-spherical structures have lower energy than the spherical droplets (Sharma et al., 2015).

\section{The Wigner-Seitz Cell Characteristics and Proton Fraction}

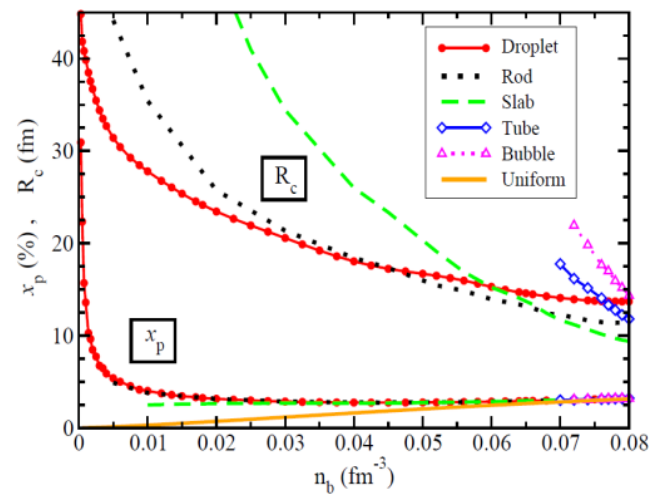

Figure 9. Radius $R_{\varepsilon}$ of the Wigner-Seitz cell and proton fraction $X_{v}=Z / A$ (given in percentage) in different geometries with respect to the baryon density (Sharma et al., 2015). 
Figure [9] displays the cell size $R_{c}$ and the proton fraction $X_{p}=Z / A$ of the equilibrium configurations against $n_{b}$ for the different shapes in Sharma's calculation. $R_{E}$ shows a nearly monotonic downward trend when the density increases, in agreement with other studies of NS inner crusts. The size of the cell $R_{0}$ has a significant dependence on the geometry of the nuclear structures, as seen by comparing $R_{\bar{c}}$ of the different shapes. In the spherical solutions, the cell radius decreases from almost $45 \mathrm{fm}$ at $n_{b}=0.0003$ $\mathrm{fm}^{-3}$ to $13.7 \mathrm{fm}$ at $n_{b}=0.08 \mathrm{fm}^{-3}$ near the transition to the core. As regards the proton fraction $X_{p}$, it takes quite similar values for the various cell geometries in the ranges of densities where we obtained solutions $X_{p}$ for the respective shapes. Beyond a density of the order of $0.02 \mathrm{fm}^{-3}$ the proton fraction $X_{p}$ shows a weak change with density and after $n_{b} \sim 0.05 \mathrm{fm}^{-3}$ it smoothly tends to the value in uniform npe matter. For the spherical droplet solutions, we find that $X_{p}$ rapidly decreases from $31 \%$ at $n_{b}=0.0003 \mathrm{fm}^{-3}$ to $\sim 3 \%$ at $n_{b}$ $=0.02 \mathrm{fm}^{-3}$. It afterward remains almost constant, presenting a minimum value of $2.75 \%$ at $n_{b}=0.045$ $\mathrm{fm}^{-3}$ and then a certain increase up to $3.2 \%$ at the last densities before the core (Sharma et al., 2015).

\section{CONCLUSIONS}

From the result it can be concluded that the cylindrical shape (Fig 4) is the energetically favoured configuration up to a crustal density of $0.076 \mathrm{fm}^{-3}$ where a second change takes place to the planar slab shape. As the density of the crustal matter increases further, the energy per baryon of tubes and bubbles becomes progressively closer to that of the slabs (Sharma et al., 2015).

The spherical droplet configuration is the energetically most favourable shape all the way up to $n_{b} \sim 0.065 \mathrm{fm}^{-3}$. When the crustal density reaches $0.065 \mathrm{fm}^{-3}$ (approximately $10^{14} \mathrm{~g} / \mathrm{cm}^{3}$ ), the nucleus occupies a significant fraction of the cell volume and it may happen that non-spherical structures have lower energy than the spherical droplets.

$R_{E}$ shows a nearly monotonic downward trend when the density increases, in agreement with other studies of NS inner crusts. The size of the cell $R_{c}$ has a significant dependence on the geometry of the nuclear structures. In the spherical solutions, the cell radius decreases from almost $45 \mathrm{fm}$ at $n_{b}=0.0003 \mathrm{fm}^{-3}$ to $13.7 \mathrm{fm}$ at $n_{b}=0.08 \mathrm{fm}^{-3}$ near the transition to the core.

Beyond a density of the order of $0.02 \mathrm{fm}^{-3}$ the proton fraction $X_{p}$ shows a weak change with density and after $n_{b} \sim 0.05 \mathrm{fm}^{-3}$ it smoothly tends to the value in uniform npe matter. For the spherical droplet solutions, we find that $X_{p}$ rapidly decreases from $31 \%$ at $n_{b}=0.0003 \mathrm{fm}^{-3}$ to $\sim 3 \%$ at $n_{b}=0.02 \mathrm{fm}^{-3}$. It afterward remains almost constant, presenting a minimum value of $2.75 \%$ at $n_{b}=0.045 \mathrm{fm}^{-3}$ and then a certain increase up to $3.2 \%$ at the last densities before the core.

\section{ACKNOWLEDGEMENTS}

This study was partially supported by Astrophysics Research Group of Universitas Gadjah Mada. We thank to all student of Physics Department of Universitas Gadjah Mada who had supported us from the first time this paper written, mainly Feni Fitrishia and Yudhistira Iriana Putra.

\section{REFERENCES}

Arya AP. 1966. Fundamental of Nuclear Physics. West Virginia University, Boston.

Caplan EM , Horowitz JC. 2017. Astromaterial Science and Nuclear Pasta. Center for Exploration of Energy and Matter and Department of Physics. Indiana University, USA.

Chamel N, Haensel P. 2008. Physics of Neutron Star Crusts.

Douchin F, Haensel P. 2000. Inner edge of neutron-star crust with SLy effective nucleon-nucleon interaction. Physics Letters B 4852000 107-114.

Glendenning NK. 2000. Compact Stars : Nuclear Physics, Particle Physics, and General Relativity, Second Edition. Springer, New York.

Gandolfi S. 2012. The equation of state of neutron star matter and the symmetry energy, 11th International Conference on Nucleus-Nucleus Collisions (NN2012). Journal of Physics: Conference Series 420 (2013) 012150.

Gandolfi S, dkk. 2013. The equation of state of neutron matter, symmetry energy, and neutron star structure. arXiv: $1307.5815 \mathrm{v} 1$.

Haensel P, Potekhin AY, Yakovlev DG. 2007. Neutron Stars 1: Equation of State and Structure. Astrophysics and Space Science Library, vol. 326. Springer, New York, U.S.A.

Hashimoto Masa-aki, dkk. 1984. Shape of Nuclei in the Crust of Neutron Star. Progress of Theoretical Physics. Vol. 71, No.2, February 1984.

Iida K, Watanabe G, Sato K. 2001. Formation of Nuclear "Pasta" in Cold Neutron Star Matter. Progress of Theoretical Physics, Vol. 106, No. 3, September 2001.

Landau L. 1931. On the Theory of Star. Physikalische Zeitschrift der Sowjetunion Vol. 1, No. 2, 285-188.

Lattimer JM, Steiner WA. 2014. Constraints on the Symmetry Energy Using the Mass-Radius Relation of Neutron Stars. arXiv:1403.1186v1

Meyerhof WE. 1967. Elements of Nuclear Physics. Stanford University. McGRAW-HILL Book Company, New York.

Potekhin AY. 2011. The Physics Of Neutron Stars. astro-ph. SR, 1235-1256.

Prakash M, dkk. 1988. Equation of State and the Maximum Mass of the Neutron Stars. Phys. Rev. Lett. 61, 22.

Providencia C, dkk. 2013. Imprint of the symmetry energy on the inner crust and strangeness content of neutron stars. arXiv:1307.1436v1.

Psonis PG, Moustakidis CCh, Massen ES. 2008. Nuclear symmetry energy effects on neutron stars properties. arXiv:nuclth/0609052v2.

Roca-Maza X, Piekarewicz J. 2008. Impact of the symmetry energy on the outer crust of nonaccreting neutron stars. Phys. Rev.C78, 025807 . 
Schneider SA, dkk. 2013. Nuclear Pasta Formation. arXiv:1307.1678v1 [nucl-th] 5 Jul 2013.

Shapiro SL, Teukolsky TA. 1983. Black Holes, White Dwarf, Neutron stars. John Wiley \& Sons Inc., New York.

Sharma KB, dkk. 2015. Unified equation of state for neutron stars on a microscopic basis. arXiv: $1506.00375 \mathrm{v} 2$

Sulistyani E T. 2015. Kajian tentang Rumus Massa Semi-Empiris Pada Bintang Neutron. Laporan Penelitian. Jurusan Fisika FMIPA UGM, Yogyakarta.
Vida a I, Providência C. 2010. Density dependence of the nuclear symmetry energy: a microscopic perspective.

Vi as X, dkk. 2017. pasta-phase transitions in the inner crust of neutron stars. Acta Physica Polonica B Proceedings Supplement, Vol. 10(2017), No.1.

Yasrina A. 2011. Nukleosistesis dan Evolusi Bintang. Skripsi. Jurusan Fisika FMIPA UGM, Yogyakarta. 